\title{
LONG PERIOD WR BINARIES
}

\author{
K. ANNUK \\ W.Struve Astrophysical Observatory of Tartu \\ 202444 Tõravere \\ Estonia, USSR
}

\begin{abstract}
Now it is clear that there exist some long period (periods exceed a year) Wolf-Rayet binaries. This paper presents the results of our radial velocity study for three WR binaries: HD193077 (WN6 + O9), HD193793 (WC7 + O5-6) and HD192641 (WC7 + O9). Our spectroscopic observations confirm that HD193077 and HD193793 are long period systems with the periods of 1538 and 2886 days. Most likely, HD192641 is also a long period WR binary ( $\mathrm{P} \geq 4400$ days).
\end{abstract}

\section{Introduction}

Radial velocity studies of HD193077 were previously made by Bracher (1966), Massey (1980) and Lamontagne et al. (1982). Both Bracher and Massey have not found periodic radial velocity variations. However, Lamontagne et al. (1982), combining their own observations with the data by Bracher and Massey suggested that HD193077 is a triple system with periods of 1763 and 2.3238 days. Adding a new observation set spanning over 7 years, Annuk (1990) showed that HD193077 is still a binary system with a period of 1538 days.

Duplicity of HD193793 was first found from the infrared photometry by Williams et al. (1987). They also demonstrated that radial velocities of absorption lines varied with a period of 7.9 years $=2886$ days. A more detailed analysis of radial velocities by Moffat et al. (1987) confirmed this period. Recently, Williams et al. (1990) found from additional infrared observations a new period of $2900 \pm 10$ days.

Analogically to HD193793, infrared photometry of HD192641 shows a periodic behaviour with a possible period of 12 years $=4400$ days (Williams, van der Hucht and The, 1987). Previous insufficient radial velocity studies (Massey, Conti and Niemela, 1981; Moffat et al., 1986) have not given any periods.

\section{Observation and reduction}

The spectroscopic observations were carried out at the Tartu Observatory in the years $1980 \div 90$ using the $1.5 \mathrm{~m}$ telescope with Cassegrain spectrographs UAGS (in

K. A. van der Hucht and B. Hidayat (eds.),

Wolf-Rayet Stars and Interrelations with Other Massive Stars in Galaxies, 245-250.

(C) 1991 IAU. Printed in the Netherlands. 
the years $1980 \div 82$ ) or ASP-32 (in the years $1982 \div 90$ ) at a reciprocal dispersion of $44 \AA / \mathrm{mm}$ and $37 \AA / \mathrm{mm}$ in the blue. The plates IIaO were used.

All plates were traced on the PDS 1010 series microdensitometer at the Tartu Observatory in the photographic density mode with a $10 \mu m$ wide slit and $5 \mu m$ sampling interval.

Radial velocities of the emission and absorption lines were determined by fitting the Gaussian profiles upon the normalized intensity profiles by the method of least squares. Radial velocities of the strongest emission lines were also computed by the method of bisector. In this case the line profile is sliced up horizontally with a constant intensity step $(\Delta I=0.05)$ previously normalizing the central line intensities to unity. Radial velocities were calculated for every cut.

\section{Radial velocities and orbital parameters}

\subsection{HD193077}

We measured the radial velocities of six emission lines: NIV $\lambda 4057$, HeII $\lambda 4686$, NV $\lambda 4604$, HeII $\lambda 4541$, HeII $\lambda 4200$ and NIII $\lambda 4097$. Two stronger and symmetric lines (NIV $\lambda 4057$ and HeII $\lambda 4686$ ) were measured by the method of bisector as well. Radial velocities calculated as a mean of levels $I=0.10 \div 0.95$ do not differ substantially from those determined by a Gaussian profile fitting routine.

The absorption lines are too broad and shallow. For this reason the measurement of radial velocities of these lines is very inaccurate and here we do not discuss them.

TABLE 1. Orbital elements of HD193077

\begin{tabular}{lcccccc}
\hline \hline & NIV & HeII & NV & HeII & NIII & HeII \\
Element & $\lambda 4057$ & $\lambda 4686$ & $\lambda 4604$ & $\lambda 4541$ & $\lambda 4097$ & $\lambda 4200$ \\
\hline P (day) & 1538 & & & & & \\
$\gamma(\mathrm{km} / \mathrm{s})$ & -96.0 & 70.1 & 59.6 & 73.2 & 160.0 & 79.1 \\
& \pm 1.3 & \pm 1.2 & \pm 2.3 & \pm 2.7 & \pm 3.1 & \pm 4.8 \\
$\mathrm{~K}(\mathrm{~km} / \mathrm{s})$ & 30.6 & 32.1 & 31.8 & 34.3 & 48.5 & 41.6 \\
& \pm 1.9 & \pm 1.9 & \pm 4.0 & \pm 4.0 & \pm 4.0 & \pm 7.1 \\
$\mathrm{e}$ & 0.29 & 0.14 & 0.35 & 0.30 & 0.30 & 0.30 \\
& \pm 0.05 & \pm 0.06 & \pm 0.11 & & & \\
$\boldsymbol{\omega}\left({ }^{\circ}\right)$ & 271 & 239 & 207 & 278 & 344 & 271 \\
& \pm 12 & \pm 22 & \pm 18 & \pm 23 & \pm 17 & \\
$T_{o}(2445000+)$ & 284 & 235 & 110 & 328 & 540 & 238 \\
& \pm 39 & \pm 93 & \pm 59 & \pm 86 & \pm 63 & \pm 28 \\
$\mathrm{a} * \operatorname{sini}\left(\mathrm{R}_{\odot}\right)$ & 890 & 967 & 905 & 994 & 1406 & 1207 \\
& \pm 54 & \pm 58 & \pm 114 & \pm 116 & \pm 115 & \pm 205 \\
$\mathrm{f}(\mathrm{m})\left(\mathrm{M}_{\odot}\right)$ & 4.01 & 5.13 & 4.22 & 5.60 & 15.82 & 10.01 \\
& \pm 0.74 & \pm 0.92 & \pm 1.60 & \pm 1.96 & \pm 3.90 & \pm 5.09 \\
$\sigma(\mathrm{km} / \mathrm{s})$ & 12.65 & 11.74 & 16.78 & 21.05 & 24.73 & 33.66 \\
\hline
\end{tabular}

In order to determine the period, we used the period search programmes written 
by Pelt (1980) which are based on the technique of the frequency analysis of temporal series. Combining our velocity measurements with those of Massey (1980), Lamontagne et al. (1982) and Bracher (1966), we analyse the lines NIV $\lambda 4057$ and HeII $\lambda 4686$ and their superposition. The period was found to be about 1538 days.

Orbital elements were computed for each set of lines. They are given in Table 1. Using these elements, theoretical radial velocity curves of the NIV $\lambda 4057, \mathrm{HeII}$ $\lambda 4686$ and NV $\lambda 4604$ lines are compared with the phase data in Figure 1.

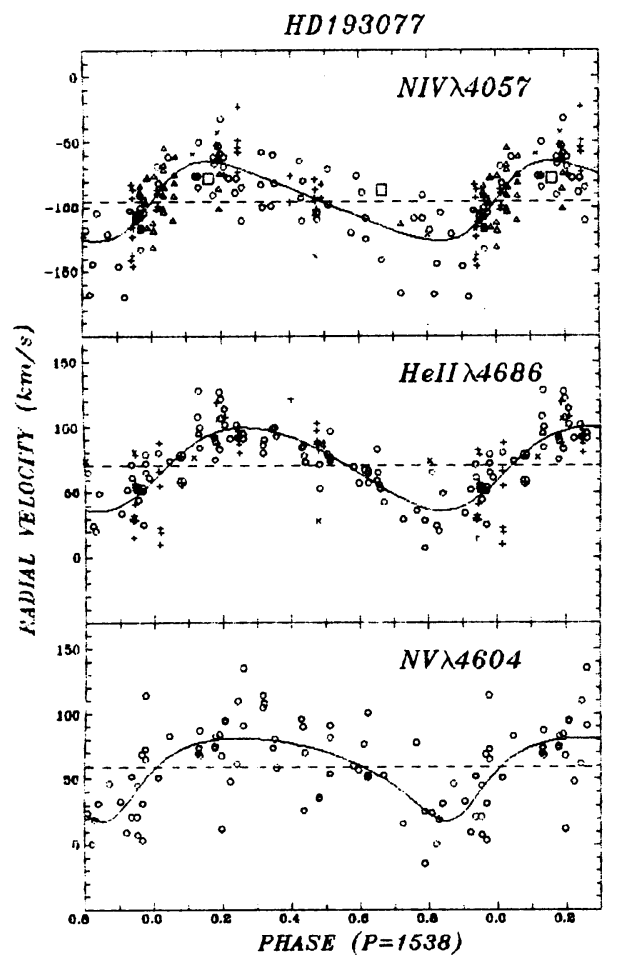

Fig. 1. Velocity curves for HD193077. The symbols have the following meanings: 0 - from this paper; + - from Lamontagne et al. (1982); $\triangle$ - from Massey (1980); $\times$ - individual plates measured by Lamontagne et al. (1982); $\square$ - Bracher's mean velocities referenced by Massey (1980).

Using the derived elements of four emission lines (NIV $\lambda 4057, \mathrm{HeII} \lambda 4686, \mathrm{NV}$ $\lambda 4604$ and HeII $\lambda 4541$ ), we conclude that $\mathrm{K}(\mathrm{em})=32 \mathrm{~km} / \mathrm{s}$ and $\mathrm{e}=0.3$. These values yield the mass function $f(m)=4.54 \mathrm{M}_{\odot}$ and the projected semi-major axis $\mathrm{a}(\mathrm{WR}) * \operatorname{sini}=930 \mathrm{R}_{\odot}$.

The mass ratio, $q=M_{W R} / M_{O}$, of other known $W R$ binaries varies between $0.26 \div$ 0.83. Assuming the mass ratio for HD193077 to be located in this range, we can estimate the minimum masses of the components: $M_{W R} \approx(1.9 \div 12.6) M_{\odot}$ and $\mathrm{M}_{\mathrm{O}} \approx(7.2 \div 15.2) \mathrm{M}_{\odot}$.

\subsection{HD193793}

In the blue region of the spectrum of HD193793 the emission line CIV $\lambda 4650$ is 
the strongest and best measurable. We measured the radial velocities of this line by the method of bisector. As CIV $\lambda 4650$ is blended in red with the emission line HeII $\lambda 4686$, we calculated the radial velocities of CIV $\lambda 4650$ as a mean of levels $I=0.55 \div 0.80$, where the blending effect is small.

Radial velocities of absorption are determined as a mean of the Balmer lines $\left(H_{\beta}\right.$, $H_{\gamma}$ and $H_{\delta}$ ) and some helium lines (HeI $\lambda 4471, \mathrm{HeI} \lambda 4026$, HeII $\lambda 4541$ and HeII $\lambda 4200)$.

TABLE 2. Orbital elements of HD193793

\begin{tabular}{lcc}
\hline \hline Element & \multicolumn{1}{c}{ abs } & CIV $\lambda 4650$ \\
\hline $\mathrm{P}($ day $)$ & 2886 & \\
$\mathrm{e}$ & $0.85 \pm 0.02$ & 0.85 \\
$\gamma(\mathrm{km} / \mathrm{s})$ & $-5.8 \pm 0.6$ & $281.5 \pm 2.9$ \\
$\mathrm{~K}(\mathrm{~km} / \mathrm{s})$ & $34.7 \pm 2.1$ & $74.0 \pm 11.9$ \\
$\omega\left({ }^{\circ}\right)$ & $34.0 \pm 3.5$ & $214=34+180$ \\
$T_{\mathrm{o}}(2446000+)$ & $120 \pm 5$ & $118 \pm 20$ \\
$\mathrm{a} * \operatorname{sini}^{\left(\mathrm{R}_{\odot}\right)}$ & $1042 \pm 63$ & $2224 \pm 357$ \\
$\mathrm{~m} * \sin ^{3} \mathrm{i}\left(\mathrm{M}_{\odot}\right)$ & $38.3 \pm 14.6$ & $18.0 \pm 4.3$ \\
$\mathrm{f}(\mathrm{m})\left(\mathrm{M}_{\odot}\right)$ & $1.83 \pm 0.33$ & $17.78 \pm 8.57$ \\
$\sigma(\mathrm{km} / \mathrm{s})$ & 8.55 & 32.77 \\
\hline
\end{tabular}

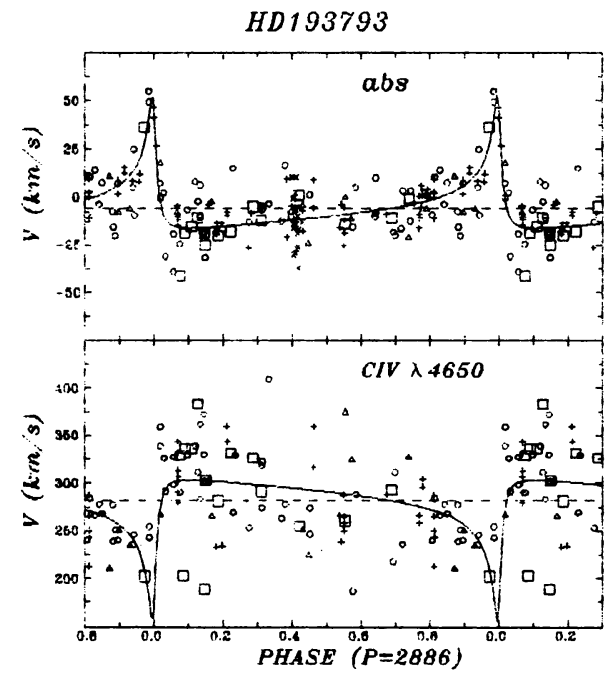

Fig. 2. Velocity curves for HD193793. The symbols have the following meanings: 0 - from this paper; + - from Conti et al. (1984); $\square$ - from Lamontagne et al. (1984); $\triangle$ - from Moffat et al. (1987); $x$ - from Galkina (1970).

We adopted the period of 2886 days as it was found by Williams et al. (1987). The period of 2900 days found recently by Williams et al. (1990) gave a somewhat 
worse solution. To combine all available observations with our own observations we made some corrections to radial velocities. In the case of emission lines we added to the data of Lamontagne et al. (1984) $+30 \mathrm{~km} / \mathrm{s}$; Conti et al. (1984) $-25 \mathrm{~km} / \mathrm{s}$ and Moffat et al. (1987) $+35 \mathrm{~km} / \mathrm{s}$. In the case of absorption lines we corrected only the data of Conti et al. (1984) with $-5 \mathrm{~km} / \mathrm{s}$ and of Galkina (1970) with -20 $\mathrm{km} / \mathrm{s}$. Such corrections are justified by the fact that different authors used different methods in determining the radial velocities.

Orbital elements are given in Table 2 and the velocity curves are shown in Figure 2. Our orbital elements are very close to those found by Williams et al. (1990). A mass ratio $\mathrm{q}=\mathrm{M}_{\mathrm{WR}} / \mathrm{M}_{\mathrm{O}} \approx 0.47$ is typical for late-type $\mathrm{WC}$ stars.

\subsection{HD192641}

The emission spectrum of HD192641 is very similar to the spectrum of HD193793. The best line for radial velocity determination is also CIV $\lambda 4650$. Radial velocities were measured by the method of bisector as a mean of levels $I=0.45 \div 0.70$.

The absorption lines are weak but not so weak that they cannot be measured. We were able to measure some absorption lines $\left(H_{\beta}, H_{\delta}, \mathrm{HeI} \lambda 4471\right.$ and partially $H_{\gamma}, \mathrm{HeI} \lambda 4026$ and HeI $\left.\lambda 4143\right)$.

Our radial velocities of the emission line CIV $\lambda 4650$ indicate a clear long-time variation. Although the scatter of radial velocities of absorption is quite large, the antiphase variation with emission line can be detected.

Direct establishment of the period from radial velocities was not available, however, from infrared photometry we may predict the period of about 4400 days or more. Such a period arises also from radial velocities, although so far the observation sets covered a little less than 4400 days.

The preliminary orbital elements are given in Table 3 . The velocity curves and an infrared photometry curve are shown in Figure 3. More observations would be needed for this star.

TABLE 3. Orbital elements of HD192641

\begin{tabular}{lcc}
\hline \hline Element & CIV $\lambda 4650$ & \multicolumn{1}{c}{ abs } \\
\hline $\mathrm{P}($ day $)$ & 4400 & \\
$\mathrm{e}$ & $0.16 \pm 0.04$ & 0.16 \\
$\gamma(\mathrm{km} / \mathrm{s})$ & $88.4 \pm 0.8$ & $-6.6 \pm 3.0$ \\
$\mathrm{~K}(\mathrm{~km} / \mathrm{s})$ & $26.3 \pm 1.1$ & $25.0 \pm 3.7$ \\
$\omega\left({ }^{\circ}\right)$ & $142.0 \pm 14.7$ & $322=142+180$ \\
$T_{\mathrm{o}}(2447000+)$ & $441 \pm 164$ & 441 \\
$\mathrm{a} * \operatorname{sini}^{\left(\mathrm{R}_{\odot}\right)}$ & $2259 \pm 98$ & $2156 \pm 314$ \\
$\mathrm{~m} * \sin ^{3} \mathrm{i}\left(\mathrm{M}_{\odot}\right)$ & $28.9 \pm 9.7$ & $30.4 \pm 7.0$ \\
$\mathrm{f}(\mathrm{m})\left(\mathrm{M}_{\odot}\right)$ & $8.01 \pm 1.04$ & $6.97 \pm 3.04$ \\
$\sigma(\mathrm{km} / \mathrm{s})$ & 6.56 & 19.15 \\
\hline
\end{tabular}




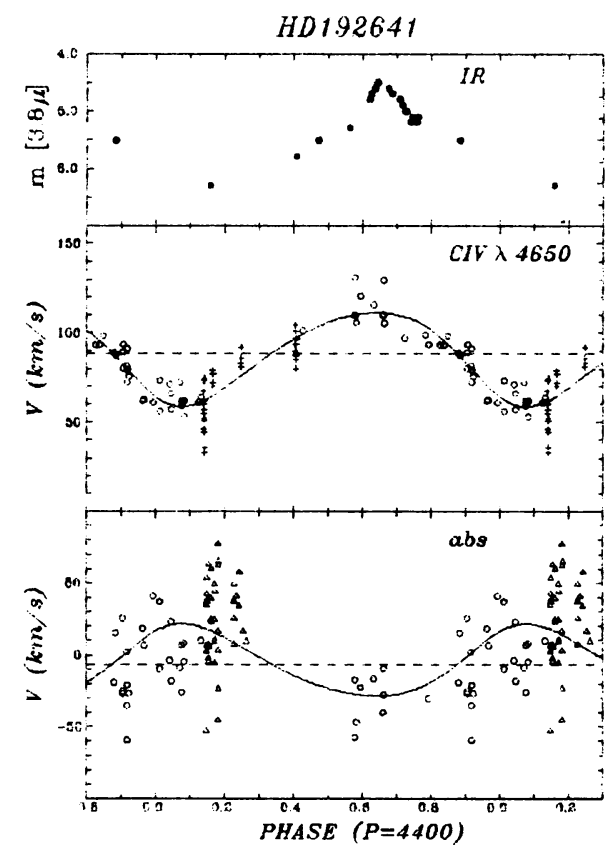

Fig. 3. Velocity curves and an infrared curve for HD192641. The symbols have the following meanings: * - from this paper; + - from Moffat et al. (1986); $\triangle$ - from Massey, Conti and Niemela (1981). Infrared photometry based on the paper of Williams, van der Hucht and The (1987).

\section{References}

Annuk, K., 1990, Acta Astronomica, (in press).

Bracher, K., 1966, Thesis Indiana University.

Conti, P.S., Roussel-Dupre, D., Massey, P. and Rensing, M., 1984, Astrophys. J., 282, 693.

Galkina, T.S., 1970, Izvestiya Krymskoi Astrof. Obs., 41-42, 283.

Lamontagne, R., Moffat, A.F.J., Koenigsberger, G. and Seggewiss, W., 1982, Astrophys. J., 253, 230.

Lamontagne, R., Moffat, A.F.J. and Seggewiss, W., 1984, Astrophys. J., $277,258$.

Massey, P., 1980, Astrophys. J., 236, 526.

Massey, P., Conti, P.S. and Niemela, V.S., 1981, Astrophys. J., 246, 145.

Moffat, A.F.J., Lamontagne, R., Shara, M.M. and McAlister, H.A., 1986, Astron. J., $91,1392$.

Moffat, A.F.J., Lamontagne, R., Williams, P.M., Horn, J. and Seggewiss, W., 1987, Astrophys. J., 312, 807.

Pelt, J., 1980, Frequency analysis of astronomical temporal series, (Valgus, Tallinn).

Williams, P.M., van der Hucht, K.A. and The, P.S., 1987, Quarterly J. Roy. Astron. Soc., 28, 248.

Williams, P.M., van der Hucht, K.A., van der Woerd, H., Wamsteker, W.M., Geballe, T.R., Garmany, C.D. and Pollock, A.M.T., 1987, in: Instabilities in Luminous Early-Type Stars, eds: H. Lamers and C.de Loore, p.221.

Williams, P.M., van der Hucht, K.A., Pollock, A.M.T., Florkowski, D.R., van der Woerd, H. and Wamsteker, W.M., 1990, Mon. Not. R. Astr. Soc., 243, 662. 